\title{
Platelet Mass Distribution Width
}

National Cancer Institute

\section{Source}

National Cancer Institute. Platelet Mass Distribution Width. NCI Thesaurus. Code C132380.

The determination of the variation defined by two standard deviations of the platelet dry mass distribution in a biological sample. 